\title{
IDENTIFICAÇÃO DO RISCO DE QUEDAS EM IDOSOS APÓS ACIDENTE VASCULAR ENCEFÁLICOa
}

\author{
Identification of the risk of falls in elderly after stroke
}

\author{
Identicación del riesgo de caídas en ancianos después de accidente cerebrovascular
}

Alice Gabrielle de Sousa Costa ${ }^{1}$

Tahissa Frota Cavalcante ${ }^{4}$

\author{
Ana Railka de Souza Oliveira²
}

Rafaella Pessoa Moreira ${ }^{3}$

\section{RESUMO}

Thelma Leite de Araujo $0^{5}$

0 objetivo desse estudo foi identificar a ocorrência do diagnóstico de enfermagem Risco de quedas em indivíduos idosos acometidos por acidente vascular encefálico. Estudo do tipo exploratório, realizado em unidades de reabilitação, no período de novembro de 2007 a março de 2008, por meio de entrevista e exame físico. Utilizou-se a Taxonomia Il da Nursing American North Diagnosis Association quanto à nomeação diagnóstica. A população foi composta por 73 idosos com idade média de 69,5 anos, predominância do sexo feminino, baixo nível de escolaridade e renda financeira. Encontrou-se média de 1,6 episódio de acidente vascular encefálico em 2,4 anos. 0 diagnóstico esteve presente na totalidade dos participantes, e os fatores de risco mais percebidos foram: Força diminuída nas extremidades inferiores, Mobilidade física prejudicada, Dificuldades na marcha e Equilíbrio prejudicado. Assim, a avaliação diagnóstica contínua e individualizada faz-se imprescindível para nor tear ações preventivas aos problemas de saúde dos idosos.

Palavras-chave: Idoso. Diagnóstico de Enfermagem. Acidentes por Quedas. Acidente Vascular Cerebral.

\begin{abstract}
The aim of the study was to identify the occurrence of the nursing diagnosis Risk of falls in elderly who suffered a stroke. Exploratory study realized in rehabilitation units from November 2007 to March 2008 through an interview and physic examine. The Taxonomy II of Nursing American North Diagnosis Association was used for the nursing diagnosis nomination. The population was composed by 73 aged with an average age of 69,5 years, mostly women, beyond low school grade and low financial income. It was found an average of 1,6 stroke episodes in 2,4 years. The diagnosis was present at all participants and the risk factors more identified were: Decreased strength in the lower limbs, Impaired Physical Mobility, Gait Difficult and Balance Impaired. Thus, a continuos and individualized diagnosis evaluation is very important to improve preventive actions to health problems of elderly.
\end{abstract}

Keywords: Aged. Nursing diagnosis. Accidental falls. Stroke.

\section{Resumen}

El estudio tuvo como objetivo identificar la ocurrencia del diagnóstico de enfermería Riesgo de caídas en ancianos con enfermedad cerebrovascular. Estudio exploratorio, desarrollado en unidades de rehabilitación, de noviembre de 2007 a marzo de 2008, por medio de entrevista y examen físico. Se utilizó la Taxonomía II de la Nursing American North Diagnosis Association para la designación del diagnóstico. La populación fue compuesta por 73 ancianos con 69,5 años en media, predominancia de mujeres, bajo nivel educacional y renta financiera. Se encontró media de 1,6 episodios de enfermedad cerebrovascular en 2,4 años. El diagnóstico fue presente en la totalidad de los participantes y los factores de riesgo más percibidos fueron: Disminución de la fuerza en las extremidades inferiores, Movilidad física perjudicada, Dificultades en la marcha y Equilibrio perjudicado. La evaluación diagnóstica continua e individualizada es imprescindible para direccionar las acciones preventivas a los problemas de salud de los ancianos.

Palabras clave: Anciano. Diagnóstico de enfermería. Accidentes por caídas. Accidente cerebrovascular.

\footnotetext{
'Enfermeira. Mestranda em Enfermagem da Universidade Federal do Ceará. Fortaleza-CE. Bolsista CAPES. Brasil. E-mail: alice_gabrielle@yahoo.com.br,²Enfermeira. Mestranda em Enfermagem da Universidade Federal do Ceará. Bolsista CAPES. Fortaleza-CE. Brasil. E-mail: railkaufc@yahoo.com.br. ${ }^{3}$ Enfermeira. Doutoranda em Enfermagem da Universidade Federal do Ceará. Bolsista CNPq. Fortaleza-CE. Brasil. E-mail: rafaellapessoa@hotmail.com. ${ }^{4}$ Enfermeira. Doutoranda em Enfermagem da Universidade Federal do Ceará. Docente da Faculdade Católica Rainha do Sertão. Fortaleza-CE. Brasil. E-mail: tahissafc@yahoo.com.br. ${ }^{5}$ Doutora em Enfermagem. Docente do Curso de Graduação e Pós-Graduação em Enfermagem da Universidade Federal do Ceará. Pesquisadora CNPq. Fortaleza-CE. Brasil. E-mail: thelmaaraujo2003@yahoo.com.br
} 


\section{INTRODUÇÃO}

A ocorrência de quedas é um dos problemas de saúde enfrentados pela população idosa tanto institucionalizada como no domicilio. Vários fatores estão envolvidos neste evento, como, por exemplo, problemas na mobilidade física, capacidade funcional, visual e cognitiva. Essas alterações, por sua vez, são decorrentes do processo fisiológico e inevitável da velhice, e estão mais acentuadas quando somadas às complicações decorrentes de doenças crônico-degenerativas não transmissíveis.

Nesse contexto, insere-se o acidente vascular encefálico (AVE), patologia de alto potencial incapacitante que está mais presente nos grupos etários de 60 anos ou mais e que pode incrementar o risco para ocorrência de quedas.

Estudo realizado com idosas atendidas em serviço de atenção básica demonstrou a presença de vários diagnósticos de enfermagem, com destaque para o de Risco de quedas e seus fatores principais desencadeantes como: idade acima de 65 anos, uso de artefatos de auxílio (bengalas), dificuldades visuais e auditivas, mobilidade física prejudicada, equilíbrio prejudicado e dificuldade na marcha. ${ }^{1}$

Destaca-se, no entanto, a escassez de estudos nessa temática de saúde, com foco em pessoas idosas acometidas por acidente vascular encefálico. Tal fato suscitou a investigação do diagnóstico Risco de queda nessa clientela, no intuito de conhecer os principais fatores de risco, para que seja possivel prevenir sua ocorrência e as possíveis complicações.

0 enfermeiro, nesse contexto, deve desenvolver ações preventivas, com vistas à identificação correta e célere do diagnóstico e seus fatores de risco. Assim, as etapas de levantamento de dados e diagnóstico configuram-se como de singular importância a esse objetivo, uma vez que norteiam as ações de intervenção do profissional.

Acerca da definição de queda, alguns autores definem queda como sendo qualquer toque ao chão de forma inesperada por qualquer parte do corpo do indivíduo, com exceção da sola dos pés. ${ }^{2-3}$

Esse agravo representa, por sua vez, um grande problema para as pessoas idosas dadas as suas consequências (injúria, incapacidade, institucionalização e morte) que são resultados da combinação de alta incidência com alta suscetibilidade a lesões, redução da capacidade funcional e institucionalização precoce. ${ }^{4}$

A pessoa idosa agrupa uma gama de fatores que aumentam o risco de quedas como: osteoporose, instabilidade postural, alteração da marcha, declínio cognitivo, dificuldades visuais, auditivas e polifarmácia. Por essa razão, faz-se necessária a avaliação global e contínua desse indivíduo. 0 ambiente residencial pode também aumentar as chances de ocorrência de quedas e deve ser incluído na programação de avaliação da pessoa idosa. Presença de escadas, ausência de diferenciação de degraus e corrimãos, lluminação inadequada, tapetes soltos, obstáculos como fios elétricos e pisos mal conservados no local de circulação são alguns dos riscos comumente observados. ${ }^{4}$

Dessa forma, a alta prevalência de quedas nessa população decorre de alterações denominadas intrínsecas e extrínsecas. Os fatores intrínsecos se referem aos problemas fisiológicos, e, no caso dos pacientes acometidos por AVE, podem-se destacar as alterações sensoriomotoras como: alterações visuais, parestesia, paresia, diminuição de flexibilidade e de mobilidade, fraqueza muscular e declínio cognitivo. Já os fatores denominados de extrínsecos estão fortemente associados às dificuldades propiciadas pelo ambiente como presença de buracos, escadas e terrenos irregulares, que também constituem grande risco de quedas. ${ }^{5}$

0 acidente vascular encefálico configura-se, assim, como importante patologia, capaz de aumentar as chances de ocorrência de quedas em decorrência do relevante comprometimento físico que pode ocasionar. Destaca-se, ainda, que a maioria dos indivíduos atingidos por esse evento é idosa, o que, por si só, constitui fator de risco para queda. Grande parte das pessoas que sobrevivem a um AVE apresenta hemiplegia e possui como comprometimento mais evidente a tendência em manter-se em uma posição de assimetria postural, com distribuição de peso menor sobre o hemicorpo parético. Essa assimetria e a dificuldade de manter o controle postural impedem a orientação e estabilidade para realizar movimentos com o tronco e membros e podem ocasionar quedas. ${ }^{6-7}$

Estudo demonstra a prevalência de quedas em 34,8\% entre indivíduos idosos, dos quais $12,1 \%$ tiveram fratura como consequência, e o evento foi significativamente maior nas mulheres $(40,1 \%)$. A prevalência de quedas associou-se estatisticamente com idade avançada, sedentarismo, autopercepção de saúde considerada ruim e maior número de medicações referido para uso contínuo. ${ }^{8}$

No intuito de identificar os pacientes suscetíveis a quedas, a taxonomia da North American Nursing Diagnosis Association (NANDA) apresenta na classe Atividade/Exercício o diagnóstico de enfermagem Risco de quedas, que possui 42 fatores de risco relacionados com o indivíduo adulto e é definido como: "Suscetibilidade aumentada para quedas que podem causar dano físico". ${ }^{9}$

Em pesquisa que investigou este diagnóstico em um grupo de idosos, os fatores de risco que apresentaram maior frequência em ambos os sexos foram: história de quedas (87,5\%); idade igual ou superior a 65 anos $(70,8 \%)$; dificuldades visuais $(95,8 \%)$; uso de medicações $(75 \%)$; quarto não familiar ou pouco iluminado $(62,5 \%)$ e ausência de material antiderrapante $(95,8 \%)$. Dentre as alterações fisiológicas mais observadas, destacaram-se as oculares e as auditivas, comuns em idosos, e que somadas às demais alterações relacionadas ao envelhecimento findam por reduzir a autonomia e independência do indivíduo, ocasionando prejuízos na sua qualidade de vida. ${ }^{10}$ 
Além da consulta de enfermagem, momento em que é possível diagnosticar e identificar os fatores de risco de cada paciente, o enfermeiro deve dedicar atenção integral às pessoas idosas ao proporcionar assistência domiciliar quando necessário e atividades de educação permanente. Faz-se importante estabelecer um plano de ações com vistas a orientar o idoso, familiares e/ou cuidador sobre os fatores de risco de queda encontrados e encorajar a adaptação do ambiente para que se torne seguro e confortável, principalmente para aqueles com maior comprometimento físico, que apresentam dificuldade na marcha e necessidade de uso de dispositivos auxiliares como bengala, muletas, cadeira de rodas e de pessoas para transferilos de posições agravantes. ${ }^{4,10}$

A partir do exposto, este estudo teve como objetivo identificar a presença do diagnóstico de enfermagem Risco de quedas e seus fatores desencadeantes em pessoas idosas que vivenciaram um ou mais episódios de acidente vascular encefálico e eram acompanhadas em serviço de reabilitação física.

\section{METODOLOGIA}

Estudo exploratório, transversal, desenvolvido com uma população de 73 idosos, dos quais a maioria $(53,4 \%)$ era do sexo feminino e $56,1 \%$ eram solteiros ou viviam com companheiro. Estes idosos participavam de serviços de saúde voltados à reabilitação física, que atendiam pacientes acometidos por AVE. Os critérios de inclusão estabelecidos para participação no estudo foram os seguintes: ter apresentado pelo menos um episódio de acidente vascular encefálico, com diagnóstico confirmado por médico; estar em fase de reabilitação física e ter idade igual ou superior a 60 anos.

Quanto à coleta de dados, esta se deu preferencialmente por fonte primária, no período de novembro de 2007 a março de 2008, por meio de um formulário que contemplou os fatores de risco do diagnóstico de enfermagem Risco de quedas proposto pela Taxonomia II da Nursing American North Diagnosis Association. ${ }^{9}$ Tal formulário foi submetido a uma avaliação por dois enfermeiros especialistas no cuidado a indivíduos com acidente vascular encefálico, além de um préteste com indivíduos com tal patologia, no intuito de atestar a contemplação de todos os fatores de risco que possibilitassem inferir o diagnóstico de enfermagem em questão. Para 0 preenchimento dos dados desse instrumento, procedeu-se a realização de uma entrevista e exame físico, focado na investigação da força muscular, problemas nos pés, dificuldade na marcha e equilíbrio prejudicado. A avaliação deste último contemplou a execução de testes auxiliares como andar com um pé atrás do outro, conseguir andar em aclive e declive, teste de Romberg e teste dedo-nariz. ${ }^{11}$

A coleta de dados era realizada simultaneamente por dois pesquisadores previamente treinados. Os dados coletados individualmente eram depois discutidos na busca de concordância diagnóstica. Utilizou-se o programa Excel 2007 para organização dos dados em planilhas e análise estatística subsequente por este mesmo programa para obtenção de frequências absolutas e percentuais, assim como média e desviopadrão.

0 projeto foi aprovado por Comitê de Ética em Pesquisa da Universidade Federal do Ceará, sob o número de protocolo 211/7. Os princípios bioéticos de não maleficência, beneficência, justiça e autonomia foram respeitados em todas as etapas do estudo. Os participantes foram informados sobre os objetivos e metodologia e concordaram em participar de forma voluntária do estudo, mediante assinatura do termo de consentimento livre e esclarecido. As despesas eventuais foram arcadas pelas pesquisadoras, com apoio do órgão de fomento.

\section{RESULTADOS}

A Tabela 1 a seguir apresenta os principais dados sociodemográficos da população do estudo.

Tabela 1 - Caracterização quanto à idade, escolaridade, renda individual e per capita, número de acidente vascular encefálico e tempo de ocorrência do último episódio nos idosos do estudo, n=73. Fortaleza, 2008

\begin{tabular}{lll}
\hline Variável & Média & Desvio-padrão \\
\hline Idade & 69,5 & 6,9 \\
Escolaridade em anos de estudo & 4,9 & 4,8 \\
Renda individual, R\$ & 614,0 & 536,0 \\
Renda per capita, R\$ & 297,0 & 227,4 \\
No episódio de AVE & 1,6 & 1,3 \\
Tempo desde último AVE (anos) & 2,4 & 4,3 \\
\hline
\end{tabular}


Além de baixa escolaridade, a maioria dos idosos tinha baixa renda per capita, que se apresentou inferior à renda individual do paciente. Os indivíduos enfrentaram em média mais de um episódio de AVE, com um intervalo de mais de dois anos desde o último evento.
Ressalta-se que o diagnóstico de enfermagem Risco de quedas esteve presente em todos os participantes do estudo; dessa forma, partiu-se à identificação dos principais fatores de risco que contribuíram para a confirmação diagnóstica, apresentandos na Tabela 2.

Tabela 2 - Fatores de risco do diagnóstico de enfermagem Risco de quedas encontrados na população feminina e masculina dos participantes do estudo, $n=73$. Fortaleza, 2008

\begin{tabular}{|c|c|c|c|c|}
\hline \multirow{2}{*}{ Fatores de risco } & \multicolumn{2}{|c|}{ Feminino $\mathrm{n}=39$} & \multicolumn{2}{|c|}{ Masculino $n=34$} \\
\hline & $\mathrm{N}$ & $\%$ & $\mathrm{~N}$ & $\%$ \\
\hline Força diminuída nas extremidades inferiores & 36 & 92,3 & 32 & 94,1 \\
\hline Dificuldade na marcha & 36 & 92,3 & 30 & 88,2 \\
\hline Agentes anti-hipertensivos & 29 & 74,3 & 26 & 76,4 \\
\hline Mobilidade física prejudicada & 23 & 58,9 & 31 & 91,1 \\
\hline Equilíbrio prejudicado & 25 & 64,1 & 18 & 52,9 \\
\hline Idade acima de 65 anos & 25 & 64,1 & 16 & 47 \\
\hline Inibidores da $\mathrm{ECA}^{*}$ & 20 & 51,2 & 16 & 47 \\
\hline Dificuldades visuais & 16 & 41 & 13 & 38,2 \\
\hline Déficits proprioceptivos & 11 & 28,2 & 16 & 47 \\
\hline Diuréticos & 13 & 33,3 & 9 & 26,4 \\
\hline Uso de cadeira de rodas & 10 & 25,3 & 9 & 26,4 \\
\hline Incontinência urinária & 13 & 33,3 & 2 & 5,8 \\
\hline Uso de dispositivos auxiliares & 8 & 20,5 & 6 & 17,3 \\
\hline Imobilização & 7 & 17,9 & 6 & 17,3 \\
\hline Estado mental rebaixado & 8 & 20,5 & 4 & 11,7 \\
\hline Morar sozinho & 4 & 10,2 & 1 & 2,9 \\
\hline Prótese de membro inferior & 2 & 5,1 & 1 & 2,9 \\
\hline
\end{tabular}

*Enzima Conversora da Angiotensina

Encontrou-se média de 6,3 fatores de risco na população feminina e de 7 na população masculina. Os demais fatores relativos ao diagnóstico em questão não foram encontrados, ou foram encontrados em apenas um participante, de modo que não houve apresentação e discussão dos mesmos.

Dentre os fatores de risco encontrados, aqueles que foram percebidos em aproximadamente $50 \%$ ou mais dos participantes de ambos os sexos foram: Força diminuída nas extremidades inferiores, Mobilidade física prejudicada, Dificuldades na marcha, Agentes anti-hipertensivos, Equilíbrio prejudicado, Idade acima de 65 anos e Inibidores da ECA.

0 fator de risco Déficit proprioceptivo foi mais frequente na população masculina, enquanto Incontinência urinária,
Estado mental rebaixado e Morar sozinho foram mais presentes nas mulheres. Os demais fatores evidenciados se apresentaram equivalentes em ambos os sexos.

\section{DISCUSSÃO}

0 diagnóstico de enfermagem Risco de quedas foi apresentado pela totalidade dos participantes do estudo, que acometidos por um evento de AVE, muitas vezes apresentavam incapacidades físicas importantes, fato este que pode incrementar as chances de ocorrência do evento queda, configurando-se como um fator de risco a este agravo. ${ }^{6}$

A população do estudo foi em sua maioria composta por mulheres, fato que se configura como um fator de risco para o 
diagnóstico. Os fatores de risco intrínsecos foram os mais identificados, esses estavam relacionados principalmente às alterações na mobilidade física como: Força diminuída nas extremidades inferiores, Mobilidade física prejudicada, Dificuldades na marcha e Equilíbrio prejudicado. Contudo, é válido ressaltar que os ambientes residenciais dos pacientes não foram visitados, confiando-se no relato dos mesmos quanto aos dados relativos às condições extrínsecas de risco de queda.

As alterações clínicas e funcionais relativas à mobilidade, como a debilidade muscular, alterações da marcha e do equilibrio e a incapacidade para realizar as atividades da vida diária, estão for temente associadas a quedas. Autores destacaram ainda que, em alguns casos, essas variáveis foram identificadas concomitante à presença de doenças neurológicas, como o AVE, que incrementam o risco de quedas. ${ }^{12}$

Uma pesquisa que investigou o diagnóstico de enfermagem Mobilidade física prejudicada em pacientes com AVE, predominantemente da faixa etária acima de 60 anos, encontrou sua alta prevalência (80\%). Destacou-se o fato de que muitas das características definidoras deste diagnóstico podem ser favoráveis à ocorrência de quedas, tais como Instabilidade Postural, Movimentos não coordenados e descontrolados, Amplitude limitada do movimento, Diminuição da força muscular, entre outros. ${ }^{13}$

A diminuição da força muscular, assim como de massa corporal, pode estar relacionada à idade; esse processo é denominado sarcopenia e vem tornando-se conhecido como causa de alta morbidade e disfunção entre os idosos. A diminuição da força muscular nas extremidades inferiores, por exemplo, compromete a mobilidade e as atividades de vida diária e aumenta o risco de quedas nessa população. ${ }^{14}$

Essas alterações físicas, que prejudicam a mobilidade conduzem, por sua vez, a uma maior chance de ocorrência de quedas. Nos últimos 18 anos, os estudos realizados com a temática queda, demonstraram que os fatores de risco mais identificados foram: ter idade acima de 60 anos, ser do sexo feminino, apresentar déficit muscular, problemas na marcha, incapacidade funcional, alterações cognitivas e consumir medicação psicotrópica. ${ }^{12}$

Tais fatos corroboram os achados do estudo ora elaborado, uma vez que a população era composta por idosos com média de idade de 69,5 anos ( $\pm 6,9)$, em sua maioria do sexo feminino, e, destas, 20,5\% apresentavam estado mental rebaixado, com comprometimento cognitivo.

Vale ressaltar que, na pesquisa em questão, os déficits proprioceptivos foram mais percebidos nos participantes do sexo masculino; no entanto, não se encontrou literatura que respaldasse esse achado. Já os fatores de risco mais encontrados na população feminina como Estado mental rebaixado, Incontinência urinária e Morar sozinha, também foram mais evidenciados nesse mesmo sexo em outra pesquisa. ${ }^{10}$

A presença de incontinência urinária deve ser sempre avaliada, pois cerca de $30 \%$ das pessoas idosas não institucionalizadas costumam apresentá-la e nem sempre a referem na avaliação clínica por vergonha ou por acharem isso normal no processo fisiológico de envelhecimento. A restrição da mobilidade e os efeitos medicamentosos podem ser causas reversíveis desse problema e devem ser corretamente investigados. ${ }^{4}$

0 Estado mental rebaixado, que se traduz por limitações da função cognitiva constitui um dos maiores problemas dos pacientes idosos e capaz de incrementar o risco de ocorrência de quedas. Ademais, pode resultar em dependência e perda da autonomia, com grande sobrecarga para os familiares ecuidadores. ${ }^{15}$

As evidências supracitadas são corroboradas por outro estudo que também verificou associação estatística relevante entre a ocorrência do evento queda e a perda de equilíbrio, a pressão arterial elevada e fraqueza muscular. ${ }^{10}$

Sabe-se que a pressão arterial elevada é o principal fator de risco para complicações cardiovasculares, como o acidente vascular encefálico, e está presente em cerca de $70 \%$ dos casos desse evento. Por esse motivo, é comum pacientes portadores de AVE fazerem uso de inúmeros medicamentos como os antihipertensivos e inibidores da ECA, que podem ser propensores de quedas em função de efeitos hipotensores e possíveis interações medicamentosas. ${ }^{16}$

A doença e a polifarmácia estão presentes no cotidiano das pessoasidosas. As interações medicamentosas podem comprometer a saúde do idoso, inclusive ao desencadear a hipotensão postural que aumenta o risco de queda, de modo que se torna importante avaliar as possíveis interações medicamentosas, assim como orientar adequadamente os idosos e seus familiares. ${ }^{4}$

As dificuldades visuais apresentadas pela população geral do estudo configuram-se como outro importante facilitador da ocorrência de queda, principalmente quando em associação a outros fatores. Ressalta-se que a existência de inúmeros fatores de riscos representa-se como forte indicador à ocorrência de quedas e pode ainda comprometer 0 desempenho das atividades de vida diária dos pacientes. ${ }^{17}$

Ao saber que uma queda pode proporcionar danos físicos, tais como fraturas, escoriações e luxações, que por sua vez podem agravar o estado de saúde do paciente, é imprescindível que a equipe de saúde saiba identificar e diagnosticar os indivíduos propensos a sofrer queda, no intuito de minimizar esse grave e frequente acontecimento. ${ }^{18}$

Para o enfermeiro, avaliar a suscetibilidade para a ocorrência de quedas na população idosa que vivenciou um AVEé, assim, uma ação pertinente e necessária, uma vez que se acredita que os diagnósticos representam o foco do cuidado de enfermagem. ${ }^{10}$

Medidas práticas podem ser implementadas para minimizar a ocorrência de quedas e suas consequências danosas entre as pessoas idosas, tais como: educação para o autocuidado, utilização de dispositivos de auxílio à marcha, utilização criteriosa de medicamentos e adaptação do ambiente em que 0 indivíduo vive. Para se estabelecer um ambiente seguro às pessoas idosas, não são necessários grandes gastos ou mudanças radicais no âmbito da família. No entanto, com a 
implementação de cuidados, há uma considerável melhoria da qualidade de vida dessas pessoas. ${ }^{4}$

\section{CONCLUSÕES}

A identificação do diagnóstico de enfermagem Risco de quedas em pessoas que sofreram AVE foi pertinente, uma vez que esteve presente na totalidade dos participantes, fato que demonstra a importância de uma avaliação correta, que focalize os principais problemas de saúde enfrentados por essas pessoas.

Ser idoso, do sexo feminino, usuário de medicamentos anti-hipertensivos e apresentar déficits motores, decorrentes de eventos incapacitantes como o acidente vascular encefálico, representaram os fatores de risco mais frequentes à ocorrência de queda.

Pontua-se, então, o alto índice de dependência física apresentado por essa população, já que os fatores de risco mais identificados do diagnóstico abordado foram os intrínsecos, relacionados às alterações da mobilidade: Força diminuída nas extremidades inferiores, Mobilidade física prejudicada, Dificuldades na marcha e Equilíbrio prejudicado.

A avaliação contínua de pacientes com AVE faz-se, assim, imprescindível, no intuito de propor ações preventivas quanto à ocorrência de quedas e que foquem as necessidades individuais com uma abordagem holística do contexto em que estejam inseridos. Destaca-se a necessidade de ações multiprofissionais com vistas à melhor adequação da terapia medicamentosa, otimização das capacidadesfísicasfuncionais e promoção da saúde desses indivíduos.

Nesse âmbito, as ações preventivas voltadas a educar, orientar e empoderar o indivíduo e família enquanto protagonistas das ações de saúde merecem singular atenção dos profissionais envolvidos com o cuidar.

\section{REFERÊNCIAS}

1. Marin MJS, Cacílio LCO, Rodrigues LCR, Ricci FA, Druzian S. Diagnósticos de enfermagem de idosas carentes de um Programa de Saúde da Família (PSF). Esc Anna Nery. 2008; 12(2): 278-84.

2.Wada N, Sohmiya M, Shimizu T, Kamoto K, Shirakura K. Clinical analysis of risk factors for falls in home-living stroke patients using functional evaluation tools. Arch Phys Med Rehab. 2007; 88(12): 1601-05.

3. Sze K, Wong E, Leung HY, Woo J. Falls among: chinese stroke patients during rehabilitation. Arch Phys Med Rehab. 2001; 82: 1219-25.

4.Ministério da Saúde (BR). Secretaria de Atenção à Saúde. Departamento de Atenção à Saúde.. Envelhecimentos e saúde da pessoa idosa. Cadernos de Atenção Básica 2006; 19. (Série A: Normas e Manuais Técnicos).

5. Christofoletti G, Oliani MM, Gobbi LTB, Gobbi S, Stella F. Risco de quedas em idosos com doença de Parkinson e Alzheimer. Rev Bras Fisiot. 2006; 10(4): 429-33.

6. Ikai T, Takehara I, Nishi M, Miyano S. Dynamic postural control in patients with hemiparesis. Am J Phys Med Rehab. 2003; 82(6): 463-69.
7. Gomes BM, Nardoni GCG, Lopes PG, Godoy E. 0 efeito da técnica de reeducação postural global em um paciente com hemiparesia após acidente vascular encefálico. Acta Fisiatr. 2006; 13(2): 103-08.

8. Siqueira FV, Facchini LA, Piccini RX, Tomasi E, Thumé E, Silveira DS, et al. Prevalência de quedas em idosos e fatores associados. Rev Saude Publica. 2007; 41(5): 749-56.

9. North American Nursing Diagnosis Association- NANDA. Diagnósticos de enfermagem da NANDA: definições e classificação 2009-2011. Porto Alegre (RS): Artmed; 2009.

10. Machado TR, Oliveira CJ, Costa FBC, Araujo TL. Avaliação da presença de risco para quedas em idosos. Rev Eletr Enferm [on-line] 2009; [citado 2009 jul 06]; 11(1): 32-8: Disponivel em: http://www.fen.ufg.br/revista/ v11/n1/v11n1a04.htm.

11. PortoCC. Semiologiamédica. 5a ed. SãoPaulo(SP):Guanabara Koogan;2005.

12. GamaZAS, Gómez-Conesa A. Factores de riesgo de caídas en ancianos: revisión sistemática. Rev Saude Pública. 2008; 42(5): 946-56.

13.Costa AGS. Mobilidade física prejudicada: identificação do diagnóstico em pacientes acometidos por acidente vascular encefálico [monografia]. Fortaleza (CE): Departamento de Enfermagem, Universidade Federal do Ceará; 2008.

14. Sverzut ACM. Histopatologia do músculo esquelético no processo de envelhecimento e fundamentação para a prática terapêutica de exercícios físicos e prevenção da sarcopenia. Rev Fisiot USP. 2003; 10(1): 24-33.

15.Costa EFA, Monego ET. Avaliação Geriátrica Ampla-AGA. Rev Univ Fed Goiás [on- line] 2003; [citado 2009 ago 14]; 5(2). Disponível em: http:/ /www.proec.ufg.br/revista_ufg/idoso/aga.html.

16. Smeltzer SC, Bare BG. Tratamento de pacientes com distúrbios vasculares cerebrais. In: Smeltzer SC, Bare BG, editoras. Brunner \& Suddarth: Tratado de enfermagem médico-cirúrgica. $10^{\mathrm{a}}$ ed. Rio de Janeiro (RJ): Guanabara Koogan; 2005.

17. Lamoreux EL, Chong E, Wang JJ, Saw SM, Aung T, Mitchell P, et al. Visual impairment, causes of vision loss, and falls: The Singapore Malay Eye Study. Invest Ophthalmol Vis Sci. 2008; 49(2): 528-33.

18. RochaFLR, Marziale MHP.Prevenção de quedas em pacientes hospitalizados: cuidado de enfermagem. Acta Paul Enferm. 2001; 14(1): 62-71.

\section{NOTA}

aTrabalho desenvolvido com apoio do Conselho Nacional de Desenvolvimento Científico e Tecnológico-CNPq, dentro do projeto Identificação dos diagnósticos de enfermagem em portadores de acidente vascular encefálico nas fases aguda e pós-hospitalar, nº 474 475/2007-7. 